\title{
Desorption of excimers from the surface of solid Ne by low-energy electron or photon impact
}

\author{
Takato Hirayama, ${ }^{1, *}$ Akira Hayama, ${ }^{1}$ Takashi Adachi, ${ }^{1}$ Ichiro Arakawa, ${ }^{1,2}$ and Makoto Sakurai ${ }^{3}$ \\ ${ }^{1}$ Department of Physics, Gakushuin University, Toshimaku, Tokyo 171-8588, Japan \\ ${ }^{2}$ Institute for Molecular Science, Okazaki 444-8585, Japan \\ ${ }^{3}$ Department of Physics, Kobe University, 1-1 Rokkodai, Nada, Kobe 657-8501, Japan
}

(Received 5 August 2000; published 30 January 2001)

\begin{abstract}
If a solid $\mathrm{Ne}$ surface is irradiated by $20-200 \mathrm{eV}$ electrons or by $55-75 \mathrm{~nm}$ synchrotron radiation, $\mathrm{Ne}_{2} *$ excimers in a ${ }^{3} \Sigma_{u}$ state are desorbed to form a luminescent "plume" in front of the sample. The kinetic energy of the desorbed excimers was found to be $(0.2 \pm 0.1) \mathrm{eV}$, which indicates that the cavity ejection mechanism is valid for the excimer desorption. The decay with time of the plume emission is not of single exponential type, because the emission lifetime of the desorbed excimers, which is of the order of $10^{-6} \mathrm{~s}$, depends on their vibrational level. Most of them are in the highest vibrational level since desorption takes place much faster than vibrational relaxation.
\end{abstract}

DOI: 10.1103/PhysRevB.63.075407

PACS number(s): 78.40.Ha, 71.35.Gg, 79.20.La

\section{INTRODUCTION}

Desorption of excited species from the surface of rare-gas solids has been extensively studied for the past 10 years. ${ }^{1}$ Monochromatic synchrotron radiation has made it possible to study the detailed electronic excitation processes that lead to desorption. As to the desorption of excited atoms induced by exciton creation, two desorption mechanisms - cavity ejection (CE) and excimer dissociation (ED) - have been proposed and the experimental results have been successfully explained by these models.

Desorption of excited dimers from the surface of rare-gas solids was first suggested by Coletti et al. ${ }^{2}$ for solid Ar irradiated by a low-energy $(\leqslant 100 \mathrm{eV})$ electron beam. Reimann and co-workers ${ }^{3-5}$ systematically studied the sputtering and luminescence at the surface of solid Ar caused by MeV light ions. They detected the luminescence of the desorbed excimers by observing only the plume in front of the Ar sample using such an experimental geometry that the emission from the sample was blocked by the edge of the sample substrate. From the detailed analysis of the results, they concluded that $\mathrm{Ar}_{2} *{ }^{3} \Sigma_{u} ; 1_{u}, 0_{u}^{-}$desorbed with a kinetic energy around 0.1 $\mathrm{eV}$, and that many-body collisions during the dimerization and desorption efficiently relaxed the vibrational levels of the excimers. Excimer desorption by low-energy photons was first reported by Grigorashchenko et al. ${ }^{6}$ for solid Ar and by Savchenko et al. ${ }^{7}$ for solid Ne. Their results suggested that the desorption of excited dimers was closely related to the exciton creation.

Molecular-dynamics calculations by Johnson and coworkers have shown that the desorption of excited dimers was energetically possible for $\mathrm{Ar}_{2} *$ in ${ }^{1,3} \Sigma_{u}$ states ${ }^{8}$ and for $\mathrm{Kr}_{2} *$ in a ${ }^{1} \Sigma_{u}$ state. ${ }^{9}$ They pointed out that the excimer desorption was related to the formation of a "cavity" around a molecular-type self-trapped exciton as in the atomic desorption case. Chen et al. ${ }^{10}$ have studied the desorption of an excimer from solid $\mathrm{Ne}$ using the extended-ion method on the basis of the one-electron Hartree-Fock approximation. Their results also supported the cavity ejection mechanism in the case of excimer desorption.
Here we report our observations on the desorption stimulated by electron impact [electron stimulated desorption (ESD)] and by synchrotron radiation [photon stimulated desorption (PSD)] of excited dimers from the surface of solid Ne. In Sec. II, the experimental setup and experimental procedures will be outlined, including the description of the pinhole camera, which was used for the detection of vacuumultraviolet (VUV) emission of the excimers desorbed from the solid Ne surface. The results of the ESD and PSD experiments will be presented in Sec. III. The emission plume recorded by the pinhole camera will be identified in Sec. IV as a cloud of desorbed luminescent excimers. Finally, our experimental results will be compared with the available information on $\mathrm{Ne}_{2} *$ in Secs. V-VII. These sections contain some discussion on the vibrational levels and the kinetic energies of the desorbed excimers as well as a comparison of the desorption mechanism of excimers with that of excited atoms.

\section{EXPERIMENT}

Figure 1 gives the top view of the experimental setup, which was similar to the one we employed in a previous work $^{11,12}$ except for the addition of a pinhole camera. This was used for recording the VUV emission from the desorbed excited species forming the plume in front of the solid $\mathrm{Ne}$ sample film. The whole system was kept in a chamber evacuated to $<10^{-8} \mathrm{~Pa}$.

The sample film was prepared on a $\operatorname{Pt}(111)$ disk of $8 \mathrm{~mm}$ diameter. This was fixed to a liquid $\mathrm{He}$ cryostat and cooled down to $6 \mathrm{~K}$. The cryostat was surrounded by a liquid $\mathrm{N}_{2}$-cooled heat shield. The sample film was condensed on the Pt disk by filling the chamber with gaseous $\mathrm{Ne}$ to a pressure of $10^{-6}-10^{-4} \mathrm{~Pa}$. The film thickness was estimated from the exposure assuming the condensation coefficient to be unity.

In ESD experiments, an electron beam with an energy between 20 and $200 \mathrm{eV}$ was focused onto the sample surface. The beam current was 0.1 to a few $\mu \mathrm{A}$ depending on the 


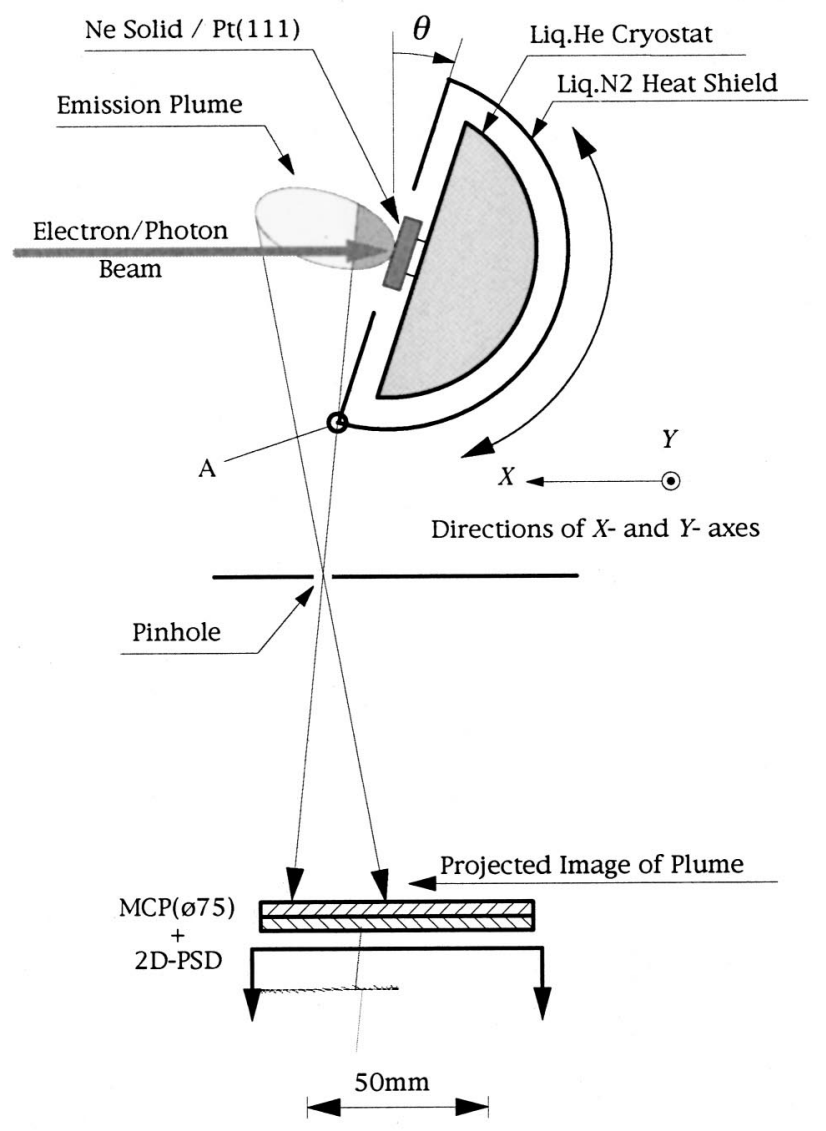

FIG. 1. Schematic diagram of the experimental setup. The elliptic region in front of the sample surface shows the emission plume due to the desorbed excited species. Emission from the gray region ("shade") in the plume does not enter the pinhole camera. The plume is projected onto the surface of an MCP. The electron and photon beams are $1 \mathrm{~mm}$ and $3 \mathrm{~mm}$ in diameter, respectively.

incident energy. The beam diameter on the sample surface was about $1 \mathrm{~mm}$.

PSD experiments were performed using the beam line BL-5B, UVSOR Facility of the Institute for Molecular Science, Okazaki. The wavelength range used in the present work was 55-75 nm, which covered the excitation energies of valence excitons in solid Ne. The number of photons incident on an area of $3 \mathrm{~mm}$ diameter on the sample surface was estimated at $(1-5) \times 10^{10} / \mathrm{s}$.

The pinhole camera consisted of a 3-mm pinhole and a microchannel plate (MCP) of $75 \mathrm{~mm}$ diameter that was equipped with a two-dimensional position-sensitive detector (2D-PSD, Quantar Technology, Inc.). It is to be noted that only the photons with wavelengths below about $150 \mathrm{~nm}$ were detectable by the MCP we employed. The sample-to-pinhole as well as the pinhole-to-MCP distance was $90 \mathrm{~mm}$, so that the magnification was unity.

In recording the spatial intensity distribution in the emission plume, we rotated the cryostat together with the heat shield so as to cut off the direct light from the sample surface by the periphery of the heat shield (marked " $A$ " in Fig. 1). The rotation angle will be denoted by $\theta$ (see Fig. 1).

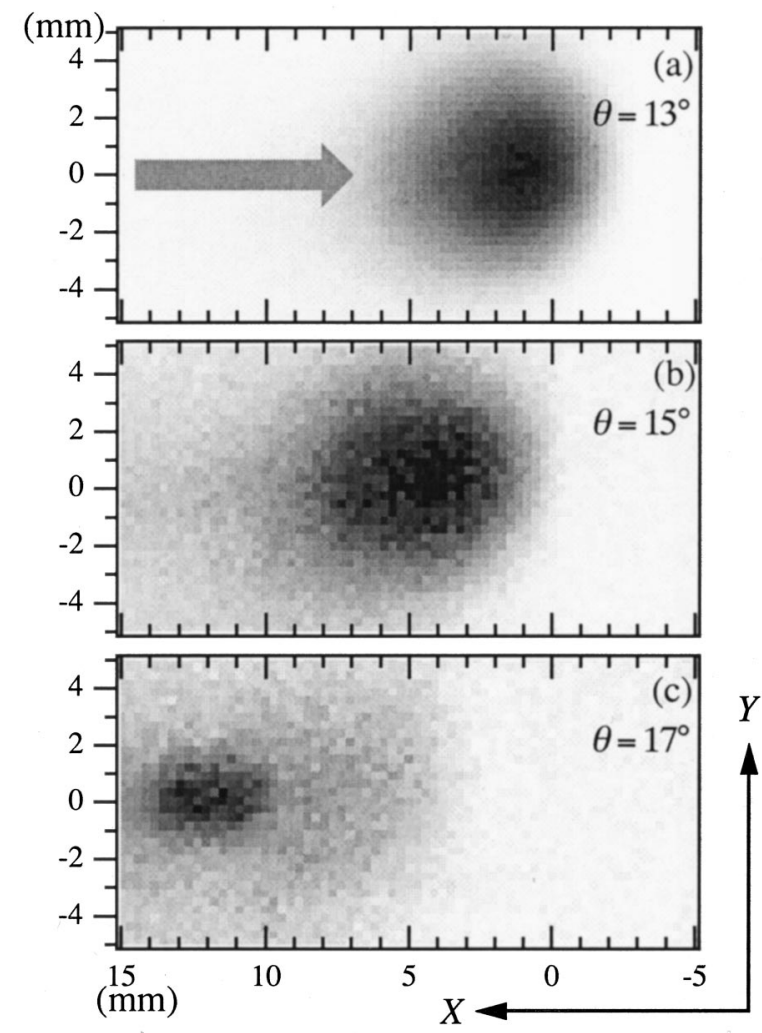

FIG. 2. Two-dimensional images of the emission from the excited species. Incident electron energy was $200 \mathrm{eV}$. (a) $\theta=13^{\circ}$, (b) $\theta=15^{\circ}$, and (c) $\theta=17^{\circ}$. The arrow in (a) shows the incident electron beam. The sample is located at $X=Y=0$. The directions of ' $X$ ' and ' $Y$ ', are designated in Fig. 1. Note that the intensity is so normalized that the maximum intensity in each picture is about the same.

\section{RESULTS}

\section{A. Electron stimulated desorption (ESD)}

\section{Spatial distribution of emission}

Figures 2(a), 2(b), and 2(c) are pinhole camera pictures for $\theta=13^{\circ}, 15^{\circ}$, and $17^{\circ}$, respectively, of the emission plume in front of the solid Ne film excited by $200-\mathrm{eV}$ electron impact. The sample film was 550 atomic monolayers (ML) thick. For convenience of description, let us adopt an orthogonal coordinate system $X, Y$ with its origin $X=Y=0$ at the center of the sample surface, the $X$ axis antiparallel to the incident electron beam, and the $Y$ axis in the vertical direction. The electron beam, $\sim 1 \mathrm{~mm}$ in diameter, was incident on the sample at $X=Y=0$ in the direction of the arrow.

In Fig. 2(a) $\left(\theta=13^{\circ}\right)$, the sample film was partly in sight of the pinhole. This resulted in the very intense signal seen near $X=Y=0$. In Figs. 2(b) and 2(c) $\left(\theta=15^{\circ}\right.$ and $\left.17^{\circ}\right)$, the direct emission from the sample was completely cut off by the heat shield periphery (A). Thus the images in Figs. 2(b) and 2(c) are due exclusively to the emission of the excited species in the plume. The signal intensities in Figs. 2(a) $-2(\mathrm{c})$ have been so normalized that the maximum intensity in each picture is about the same. 


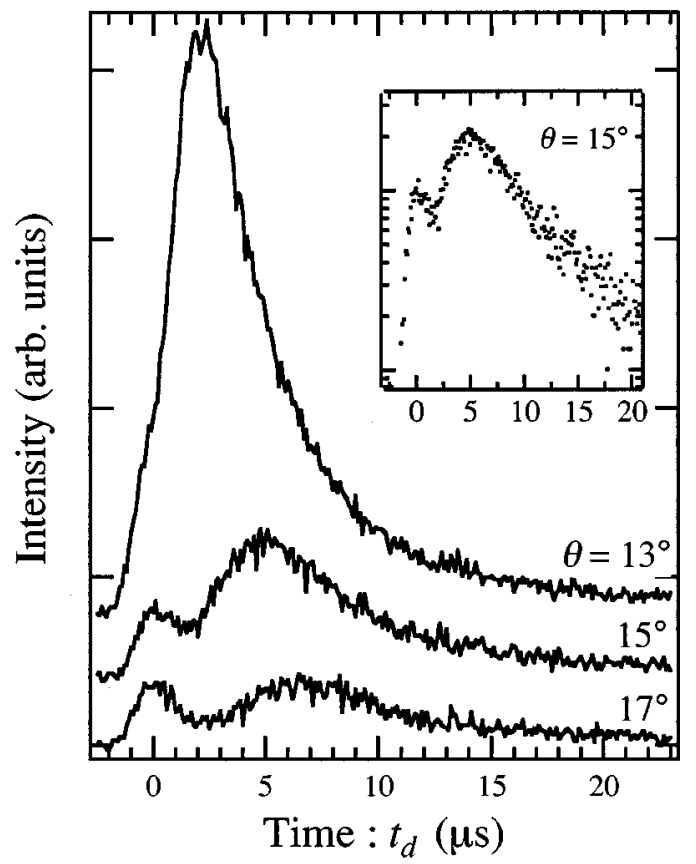

FIG. 3. Decay curves of the plume emission for $\theta=13^{\circ}, 15^{\circ}$, and $17^{\circ}$. The inset shows the curve for $\theta=15^{\circ}$ in a semilogarithmic scale. The thickness of the film was $550 \mathrm{ML}$ and the incident electron energy was $150 \mathrm{eV}$.

\section{Decay of the plume emission}

For the purpose of estimating the lifetime of the desorbed excited species, we investigated the decay with time of the plume emission. In the case of ESD, we did this pulsing of the electron beam for the stimulation of desorption, e.g., $1 \mu \mathrm{s}$ on and $500 \mu \mathrm{s}$ off. The average current of the pulsed electron beam was $1-10 \mathrm{nA}$.

The decay curves we observed for the three cases $\theta$ $=13^{\circ}, 15^{\circ}$, and $17^{\circ}$ are given in Fig. 3. The inset presents the curve for $\theta=15^{\circ}$ in a semilogarithmic scale. The incident electron energy was $150 \mathrm{eV}$ and the thickness of the film was $550 \mathrm{ML}$. When $\theta$ exceeds $13^{\circ}$ appreciably, the emission from the sample surface is cut off by the periphery of the heat shield (marked " $A$ " in Fig. 1). In that case, we find a small peak in the earliest part of the observed intensity $I$ versus time curve [Figs. 3(b) and 3(c)]. This peak is due to the bremsstrahlung of the electron beam and a short-lifetime $\left(\sim 10^{-8} \mathrm{~s}\right)$ emission from the excited species on/in the sample film, which are reflected by chamber walls.

In discussing the decay curve, we employ a scale of time $t_{d}$ whose origin is located at the peak just mentioned. Note that in the $I$ versus $t_{d}$ curve for $\theta=13^{\circ}$, in which case the sample film was partly in sight of the pinhole camera, the peak is not found at $t_{d}=0$; instead, a small shoulder is found at $t_{d}=0$. If $\theta$ exceeds $13^{\circ}$ so that the emission from the sample surface no longer enters the camera, the $I$ versus $t_{d}$ curve will have a peak at $t_{d}=0$. The emission signal will decrease as time goes on, but increases again to reach a second maximum: at $t_{d} \approx 5 \mu \mathrm{s}$ if $\theta=15^{\circ}$ and at $t_{d} \approx 7 \mu \mathrm{s}$ if $\theta=17^{\circ}$. This luminescence is due to the desorbed excited species. The time distance between the first and second

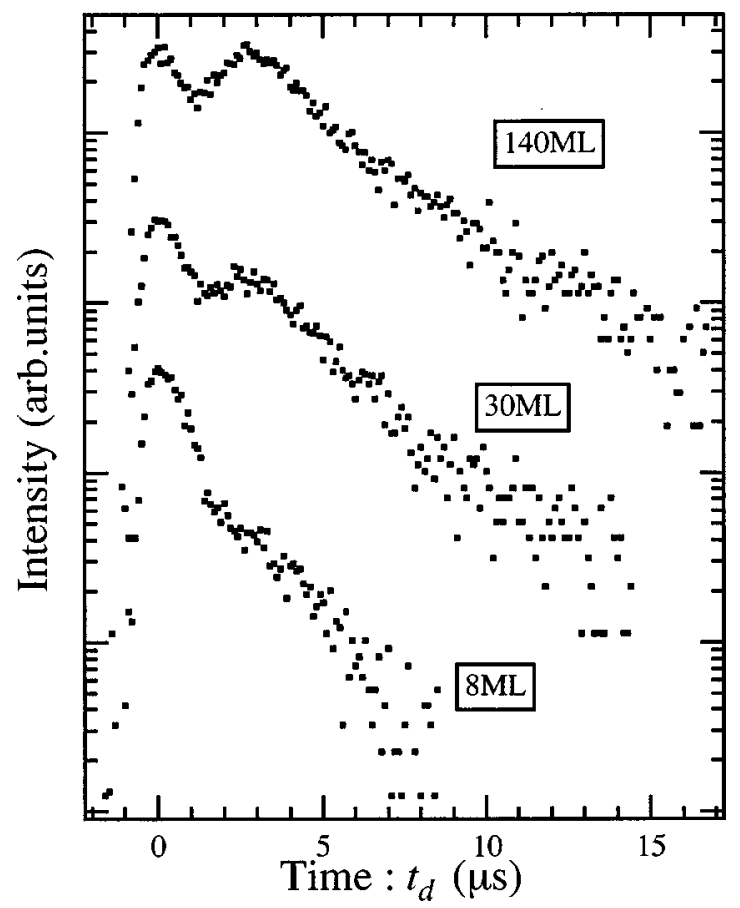

FIG. 4. Thickness dependence of the decay curve for the incident electron energy $150 \mathrm{eV}$. Sample thicknesses were $8 \mathrm{ML}, 30$ ML, and $140 \mathrm{ML}$.

maxima is roughly equal to the time required for the excited species to come out of the shade made by the heat shield (see Fig. 1).

As can be seen in the semilogarithmic plot of the decay curve (inset, Fig. 3), the decay is not of single exponential type. However, we can make a rough evaluation for the radiative lifetime $\tau$ of the excited species in the plume from the decay curves in Fig. 3. We see that the lifetime $\tau$ is of the order of $10^{-6} \mathrm{~s}$ (roughly $2 \mu \mathrm{s}<\tau<10 \mu \mathrm{s}$ ). The decay curves measured for the impact energy of $19.5 \mathrm{eV}$ (not shown), which is well below the band-gap energy of solid $\mathrm{Ne}$ $(21.6 \mathrm{eV})$, were also not of single exponential type.

To study the film thickness dependence of the decay of plume emission, we examined three films of thickness 8, 30, and $140 \mathrm{ML}$. The result (Fig. 4) indicates that the shorter lifetime species prevailed in a thin film (8 ML), while longer lifetimes are enhanced in thicker films (30 ML and $140 \mathrm{ML}$ ), indicating that the desorption of the shorter and longer lifetime species is initiated by the creation of the surface and bulk excitations, respectively. It is known that the bulk excitons diffuse to the surface efficiently in solid $\mathrm{Ne},{ }^{13}$ and contribute to the desorption of excited species. A further increase in thickness did not change the overall feature of the decay curves.

\section{B. Photon stimulated desorption (PSD)}

In PSD experiments using synchrotron radiation as the excitation source, we could also obtain pinhole camera images (not shown) like those in Fig. 2. However, measurement of the decay with time was impractical in PSD experiments because of very low signal intensities. 


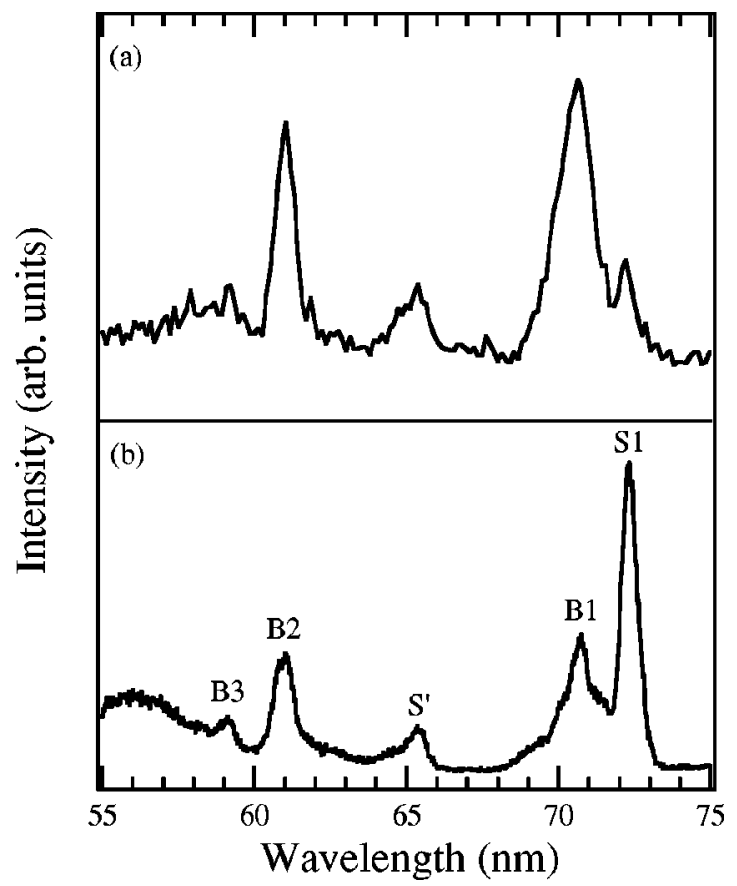

FIG. 5. (a) Plume emission intensity, or the desorption yield of $\mathrm{Ne}_{2} *\left({ }^{3} \Sigma_{u}\right)$ from the surface of solid $\mathrm{Ne}$ vs the wavelength of stimulating light. (b) Yield of excited $\mathrm{Ne}$ atoms $\mathrm{Ne}^{*}$ in ${ }^{3} P_{0,2}$ states that are desorbed from the surface of solid $\mathrm{Ne}$ through the cavity ejection mechanism (Ref. 11).

The dependence of the plume intensity on the wavelength of the incident light is shown in Fig. 5(a). Figure 5(b), which shows the desorption yield of excited $\mathrm{Ne}$ atoms $\left(\mathrm{Ne}^{*}\right)$ in ${ }^{3} P_{0,2}$ states via a cavity ejection mechanism, has been quoted from Hirayama et al. ${ }^{11}$ The peak positions correspond to the creation of the first-order surface exciton (S1), the first-, second-, and third-order bulk excitons (B1, B2, and $\mathrm{B} 3)$, and the $2 p^{5} 3 p$-type surface exciton $\left(\mathrm{S}^{\prime}\right)$, respectively. The agreement in peak positions between the $N e^{*}$ desorption yield and the plume intensity shows that the plume emission is closely related to the valence exciton creation.

The low intensities of the plume emission prevented us from studying the dependence of the size and shape of the plume on the excitation wavelength.

\section{IDENTIFICATION OF THE EXCITED SPECIES IN THE PLUME}

The fact that the radiative lifetime of the excited species in the plume is of the order of $10^{-6} \mathrm{~s}$ (Sec. III A 2) is a strong indication that the plume emission in ESD experiments is due to $\mathrm{Ne}_{2} *\left({ }^{3} \Sigma_{u}\right)$ excimers in the gas phase desorbed from the solid Ne film, whose lifetime has been evaluated at $6.6 \mu \mathrm{s}$ (Ref. 14) or $3.5 \mu \mathrm{s} \sim 11.9 \mu \mathrm{s} .{ }^{15}$ Other species that may form the plume and emit VUV photons are atoms and ions of $\mathrm{Ne}$ in excited states. However, contribution from the excited atoms can be ruled out because radiative lifetimes of the lowest excited states $\left(2 p^{5} 3 s\right)$ of a $\mathrm{Ne}$ atom in gas phase are known to be $1.65 \mathrm{~ns}$ and $20.5 \mathrm{~ns}$ for optically allowed ${ }^{1} P_{1}$ and ${ }^{3} P_{1}$ states, ${ }^{16}$ and $430 \mathrm{~s}$ and $24.4 \mathrm{~s}$ for optically forbidden ${ }^{3} P_{0}$ and ${ }^{3} P_{2}$ states, ${ }^{17}$ respectively. Emis-
TABLE I. Emission lifetimes for $\mathrm{Ne}_{2} *\left({ }^{3} \Sigma_{u}\right), \tau_{v}$ : calculated by Schneider and Cohen (Ref. 15). $\tau_{v}^{\prime}:$ present results with the correction factor $B=0.71$. See text for detail.

\begin{tabular}{crrrccccc}
\hline \hline$v$ & 0 & 1 & 2 & 3 & 4 & 5 & 6 & 7 \\
\hline$\tau_{v}(\mu \mathrm{s})$ & 11.9 & 10.2 & 8.8 & 7.7 & 6.6 & 5.6 & 4.5 & 3.5 \\
$\tau_{v}^{\prime}(\mu \mathrm{s})$ & 8.5 & 7.2 & 6.2 & 5.5 & 4.7 & 4.0 & 3.2 & 2.5 \\
\hline \hline
\end{tabular}

sion from ions is also ruled out since the plume was observed even for an excitation energy below the band-gap energy (Secs. III A 2 and III B), which also indicates that the desorption of excimers is induced by the creation of valence excitons.

Thus we are convinced that $\mathrm{Ne}$ excimers $\mathrm{Ne}_{2} *\left({ }^{3} \Sigma_{u}\right)$ are desorbed by the creation of valence excitons and emit VUV photons $[\lambda=74-81 \mathrm{~nm}$ (Ref. 18)] in vacuum forming the plume in ESD experiments (Sec. III A). This conclusion is in no way contradictory to the results of PSD experiments (Sec. III B).

\section{DISTRIBUTION OF VIBRATIONAL LEVELS OF DESORBED $\mathrm{Ne}_{2}$ *}

The observed nonsingle exponential feature of the decay curves (Sec. III A 2) can be attributed to the dependence of the emission lifetime of excimers in the gas phase on their vibrational level.

According to Schneider and Cohen's calculation, ${ }^{15}$ the lifetime of $\mathrm{Ne}_{2} *\left({ }^{3} \Sigma_{u}\right)$ varies with their vibrational level: $11.9 \mu \mathrm{s}$ for $v=0$ (ground state) and $3.5 \mu \mathrm{s}$ for $v=7$ (just below the dissociation limit). A large variation like this has been experimentally known for $A_{u}^{1}$ states of $\mathrm{Ar}_{2}{ }^{*}, \mathrm{Kr}_{2}{ }^{*}$, and $\mathrm{Xe}_{2} *{ }^{19}$

We estimate the relative populations among the vibrational levels of $\mathrm{Ne}_{2} *\left({ }^{3} \Sigma_{u}\right)$ in the following way. We assume that the intensity of the plume emission $I(t)$ consists of the contributions from all the vibrational levels $v=0$ to $v=7$ as given below,

$$
I(t)=\sum_{v=0}^{7} A_{v} \exp \left(-\frac{t}{B \tau_{v}}\right) .
$$

Here $\tau_{v}$ is the emission lifetime calculated by Schneider and Cohen (Table I), $A_{v}$ is a preexponential coefficient, and $B$ is a correction factor to provide for the uncertainty of their calculation. We can estimate $A_{v}$ and $B$ for each of the decay curves in Figs. 3 and 4 by fitting Eq. (1) to the observed curve. The relative population for the level $v$ given by

$$
\begin{gathered}
P(v)=\int_{0}^{\infty} A_{v} \exp \left(-\frac{t}{B \tau_{v}}\right) d t=A_{v} B \tau_{v}, \\
\sum_{v=0}^{7} P(v)=1,
\end{gathered}
$$

is shown in Fig. 6, and the obtained lifetimes $\tau_{v}^{\prime}\left(=B \tau_{v}\right)$ are given in Table I. As seen in Fig. 6, the relative population is 


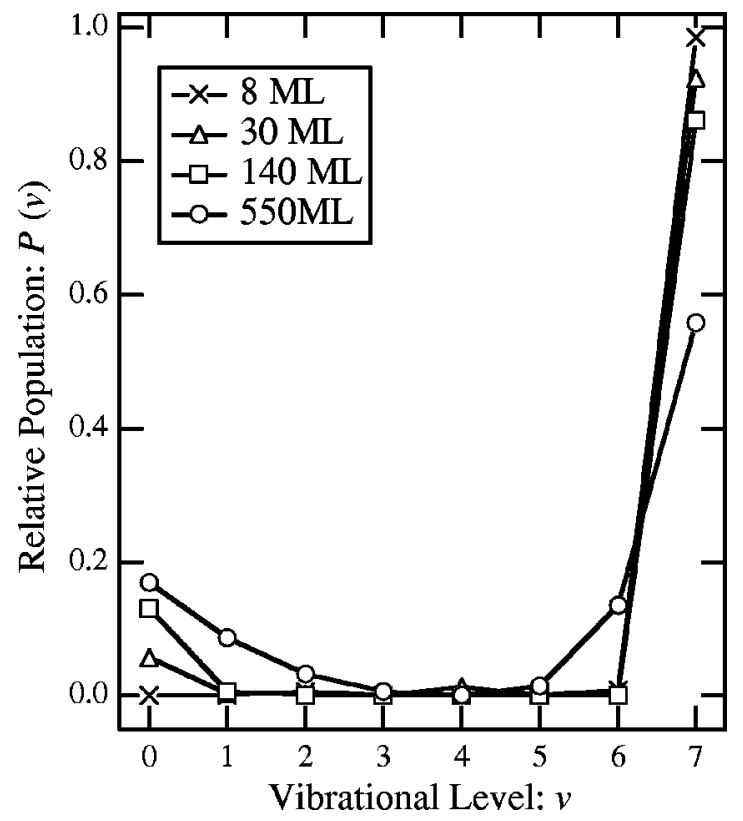

FIG. 6. Relative population $P(v)$ for desorbed excimers measured with the sample thicknesses of $8(\times), 30(\triangle), 140(\square)$, and 550(○) ML. See text for detail.

clearly non-Boltzmann: $P(v)$ for the highest vibrational level is by far the largest. This can be attributed to the fact that the time required for an excimer born on the surface to desorb $\left[\sim 10^{-12} \mathrm{~s}\right.$ (Ref. 10)] is much shorter than the time required for vibrational relaxation $\left[\sim 10^{-6} \mathrm{~s}\right.$ (Ref. 20)].

\section{KINETIC ENERGY OF DESORBED $\mathrm{Ne}_{2}{ }^{*}$}

We can estimate the average kinetic energy (KE) of desorbed $\mathrm{Ne}_{2} *$ at $0.2 \pm 0.1 \mathrm{eV}$ by combining (a) the size of the shade in the plume (Fig. 1) and (b) the time of flight of $\mathrm{Ne}_{2}{ }^{*}$ from the specimen surface to the boundary of the shade (the abscissa $t_{d}$ of the second maximum in the curves for $\theta$ $=15^{\circ}$ and $\theta=17^{\circ}$, Fig. 3). This estimation is in fair agreement with Chen et al.'s value $0.23 \mathrm{eV}$ (Ref. 10) theoretically obtained for $\mathrm{Ne}_{2}$ * desorbed from the (100) face of solid Ne. These low values of kinetic energy suggest that the cavity ejection model is plausible.

Reimann et al. ${ }^{5}$ and Chen et al. ${ }^{10}$ have studied the dependence of KE of desorbed excimers on their vibrational levels; we regret that no information along these lines can be obtained from our results.

\section{COMPARISON WITH EXCITED ATOM DESORPTION}

The dependence of the desorption yield of $\mathrm{Ne}_{2}{ }^{*}$, or the plume intensity, on the wavelength of the incident light [Fig. $5(\mathrm{a})]$ shows that the desorption yield at the first-order surface exciton (S1) excitation is much smaller than those at bulk exciton $(\mathrm{B} 1, \mathrm{~B} 2)$ excitations. In the case of the excited atom desorption [Fig. 5(b)], on the other hand, the S1 peak is much higher than the bulk peaks B1 and B2. This is probably because excimer formation on the surface becomes difficult owing to the very fast $\left[\sim 10^{-12} \mathrm{~s}\right.$ (Ref. 10) $]$ desorption of excited atoms present on the surface.

It is notable that in ESD the desorption yield of excimers induced by surface exciton creation seems to be much higher than that due to the bulk exciton creation (thickness dependence described in Sec. III A 2), in contrast to the PSD results mentioned above. This is simply due to the difference in penetration depth into solid $\mathrm{Ne}$ of photons and electrons: A photon with an energy corresponding to $\mathrm{B} 1$ exciton excitation will penetrate about $3 \mathrm{~nm}(\sim 11 \mathrm{ML})$ into solid $\mathrm{Ne}^{21}$ while an electron with a kinetic energy around $100 \mathrm{eV}$ will penetrate less than $1 \mathrm{~nm},{ }^{22}$ creating an exciton preferentially near the surface.

\section{SUMMARY}

We found that when the surface of solid $\mathrm{Ne}$ is excited by electron or photon impact, valence excitons are created and $\mathrm{Ne}$ excimers $\mathrm{Ne}_{2} *\left({ }^{3} \Sigma_{u}\right)$ are desorbed to form a luminescent plume. This, we believe, is the first systematic report on excimer desorption from the solid rare-gas surfaces purely induced by valence exciton creation.

The decay of the plume emission is not of single exponential type, owing to the dependence of the emission lifetime on the vibrational level of the excimers. Most of the desorbed excimers are in the highest vibrational level, showing that the desorption process is much faster than vibrational relaxation.

The kinetic energy of desorbed excimers is estimated at $0.2 \pm 0.1 \mathrm{eV}$. This shows that the excimer desorption takes place through the cavity ejection mechanism.

\section{ACKNOWLEDGMENTS}

The authors are indebted to Dr. Elena V. Savchenko in Verkin Institute for Low Temperature Physics, Ukraine, for her valuable discussions and comments. We would also like to thank Professor Emeritus Koreo Kinosita, Gakushuin University, for his critical reading of the manuscript. This work was partly supported by a Grant-in-Aid for Scientific Research from the Ministry of Education, Science, Sports, and Culture, Japan.
*Corresponding author. FAX: +81-3-3987-6732; Email address: takato.hirayama@gakushuin.ac.jp

${ }^{1}$ I. Arakawa, Molecular Crystals and Liquid Crystals (Gordon and Breach Science Publishers, New York, 1998), Vol. 314, pp. 4758.

${ }^{2}$ F. Coletti, J. Debever, and G. Zimmerer, J. Phys. (France) Lett. 45, L467 (1984).
${ }^{3}$ C. Reimann, W. Brown, and R. Johnson, Phys. Rev. B 37, 1455 (1988).

${ }^{4}$ C. Reimann, W. Brown, M. Nowakowski, S. Cui, and R. Johnson, in Desorption Induced by Electronic Transitions, DIET-IV, edited by G. Betz and P. Varga (Springer, Heidelberg, 1990), pp. 226-234.

${ }^{5}$ C.T. Reimann, W.L. Brown, D.E. Grosjean, and M.J. Nowa- 
kowski, Phys. Rev. B 45, 43 (1992).

${ }^{6}$ O. Grigorashchenko, A. Ogurtsov, E. Savchenko, J. Becker, M. Runne, and G. Zimmerer, Surf. Sci. 390, 277 (1997).

${ }^{7}$ E.V. Savchenko, T. Hirayama, A. Hayama, T. Koike, T. Kuninobu, I. Arakawa, K. Mitsuke, and M. Sakurai, Surf. Sci. 390, 261 (1997).

${ }^{8}$ S. Cui, R. Johnson, C. Reimann, and J. Boring, Phys. Rev. B 39, 12345 (1989).

${ }^{9}$ W. Buller and R. Johnson, Phys. Rev. B 43, 6118 (1991).

${ }^{10}$ L.F. Chen, G.Q. Huang, and K.S. Song, Nucl. Instrum. Methods Phys. Res. B 116, 61 (1996).

${ }^{11}$ T. Hirayama, A. Hayama, T. Koike, T. Kuninobu, I. Arakawa, K. Mitsuke, M. Sakurai, and E. Savchenko, Surf. Sci. 390, 266 (1997).

${ }^{12}$ T. Kuninobu, A. Hayama, T. Hirayama, and I. Arakawa, Surf. Sci. 390, 272 (1997).
${ }^{13}$ N. Schwentner, E. E. Koch, and J. Jortner, Electronic Excitations in Condensed Rare Gases, Springer Tracts in Modern Physics, Vol. 107 (Springer-Verlag, Berlin, 1985).

${ }^{14}$ T. Oka, K.R. Rao, J. Redpath, and R. Firestone, J. Chem. Phys. 61, 4740 (1974).

${ }^{15}$ B. Schneider and J.S. Cohen, J. Chem. Phys. 61, 3240 (1974).

${ }^{16}$ N.D. Bhaskar and A. Lurio, Phys. Rev. A 13, 1484 (1976).

${ }^{17}$ N.E. Small-Warren and L.Y. Chow Chiu, Phys. Rev. A 11, 1777 (1975).

${ }^{18}$ J.S. Cohen and B. Schneider, J. Chem. Phys. 61, 3230 (1974).

${ }^{19}$ A. Madej and B. Stoicheff, Phys. Rev. A, 38, 3456 (1988).

${ }^{20}$ F. Coletti, J. Debever, and G. Zimmerer, J. Chem. Phys. 83, 49 (1985).

${ }^{21}$ D. Pudewill, F.J. Himpsel, N. Schwentner, M. Skibowski, E.E. Koch, and J. Jortner, J. Chem. Phys. 65, 5226 (1976).

${ }^{22}$ N. Schwentner, Phys. Rev. B 14, 5490 (1976). 